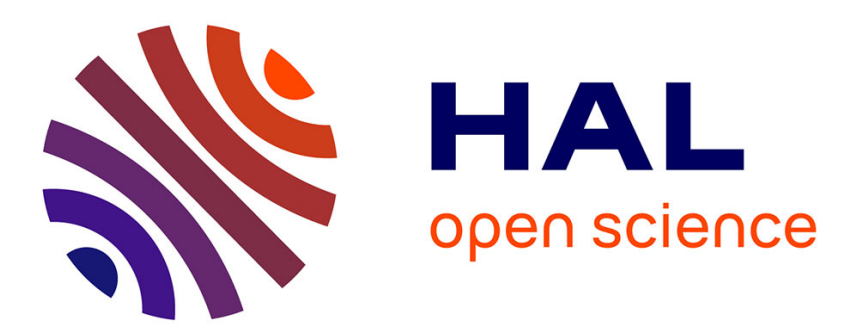

\title{
Une décharge sauvage à Port-au-Prince: une confrontation méthodologique et émotionnelle à la démarche de recherche
}

Alice Corbet

\section{- To cite this version:}

Alice Corbet. Une décharge sauvage à Port-au-Prince : une confrontation méthodologique et émotionnelle à la démarche de recherche. Les Cahiers d'Outre-Mer. Revue de géographie de Bordeaux, 2020, Sociétés et espace haïtiens contemporains : nouveaux regards, 279, pp.315-326. 10.4000/com.10416 . hal-02901186

\author{
HAL Id: hal-02901186 \\ https://hal.science/hal-02901186
}

Submitted on 16 Jul 2020

HAL is a multi-disciplinary open access archive for the deposit and dissemination of scientific research documents, whether they are published or not. The documents may come from teaching and research institutions in France or abroad, or from public or private research centers.
L'archive ouverte pluridisciplinaire HAL, est destinée au dépôt et à la diffusion de documents scientifiques de niveau recherche, publiés ou non, émanant des établissements d'enseignement et de recherche français ou étrangers, des laboratoires publics ou privés. 
Corbet Alice

CNRS

LAM

«Une décharge sauvage à Port-au-Prince ; une confrontation méthodologique et émotionnelle à la démarche de recherche », Les Cahiers d'Outre-Mer, numéro 279, paru en 2020.

\section{Une décharge sauvage à Port-au-Prince :}

\section{une confrontation méthodologique et émotionnelle à la démarche de recherche}

Suite au séisme de janvier 2010 en Haïti, qui frappa particulièrement la capitale Portau-Prince, toute la dynamique urbaine de la région fut modifiée. En quelques secondes, des milliers de bâtiments s'écroulèrent. Plus d'un million de sans-abris dut trouver des solutions de relogement, que ce soit auprès de familles ou d'amis, dans des camps de fortune installés dans les interstices des villes, ou dans ceux organisés par les ONG.

Progressivement, une zone à quelques kilomètres au Nord de l'agglomération se développa. Je l'ai découverte en suivant un groupe de déplacés violemment expulsés d'un terrain vague du centre-ville qu'ils avaient nommé «Camp Mosaïque » et qu'ils occupaient en attendant un relogement (ce «déguerpissement » mis en œuvre de nuit par des hommes armés permis ensuite à une banque de s'installer). Dans une région très aride, dite "plaine de Cul-de-Sac » car entourée de mornes (monts) hostiles, un double mouvement attira les sansabris. D'une part, un immense camp créé par des ONG, dénommé Corail, provoqua un appel d'air auprès des démunis : possibilité de rachat d'aide alimentaire, espoir de bénéficier de l'aide d'associations, développement d'une économie locale, accroissement des transports, possibilité de louer ou de racheter un «T-shelter» (abris) aux habitants du camp... D'autre part, les alentours de Corail étaient vides et hostiles, mais libres et disponibles à ceux qui avaient tout perdu en ville - bien que leur statut juridique était indéterminé.

\section{Une décharge sauvage à l'écart de l'æagolormération port-au-princienne}

C'est ainsi que le quartier de Canaas: fut sréć installé entre Croix des Bouquets à l'Est, Morne-à-Cabris au Nord-Est, Titanyen au Nord-Ouest, et la route Nationale 1 (axe majeur reliant la capitale aux régions du Nord) à i'Ouest. Rapidement, la zone s'est déployée, de bicoques précaires en maisons en dur, attirant d'abcrd s,eux qui n'avaient nulle part où aller puis toutes les classes sociales. Jeux de pouvoir et d'urbanisme s'y développèrent à la façon spontanée des zones marginales laissées à elles-mémes. Attirée par ce cas fascinant de création spontanée d'une ville, que ses habitants appelèrent vite Canaun (et qui accueillerait aujourd'hui plus de 200000 habitants), je décidais d'y mener mes recherches (Corbet, 2015). 


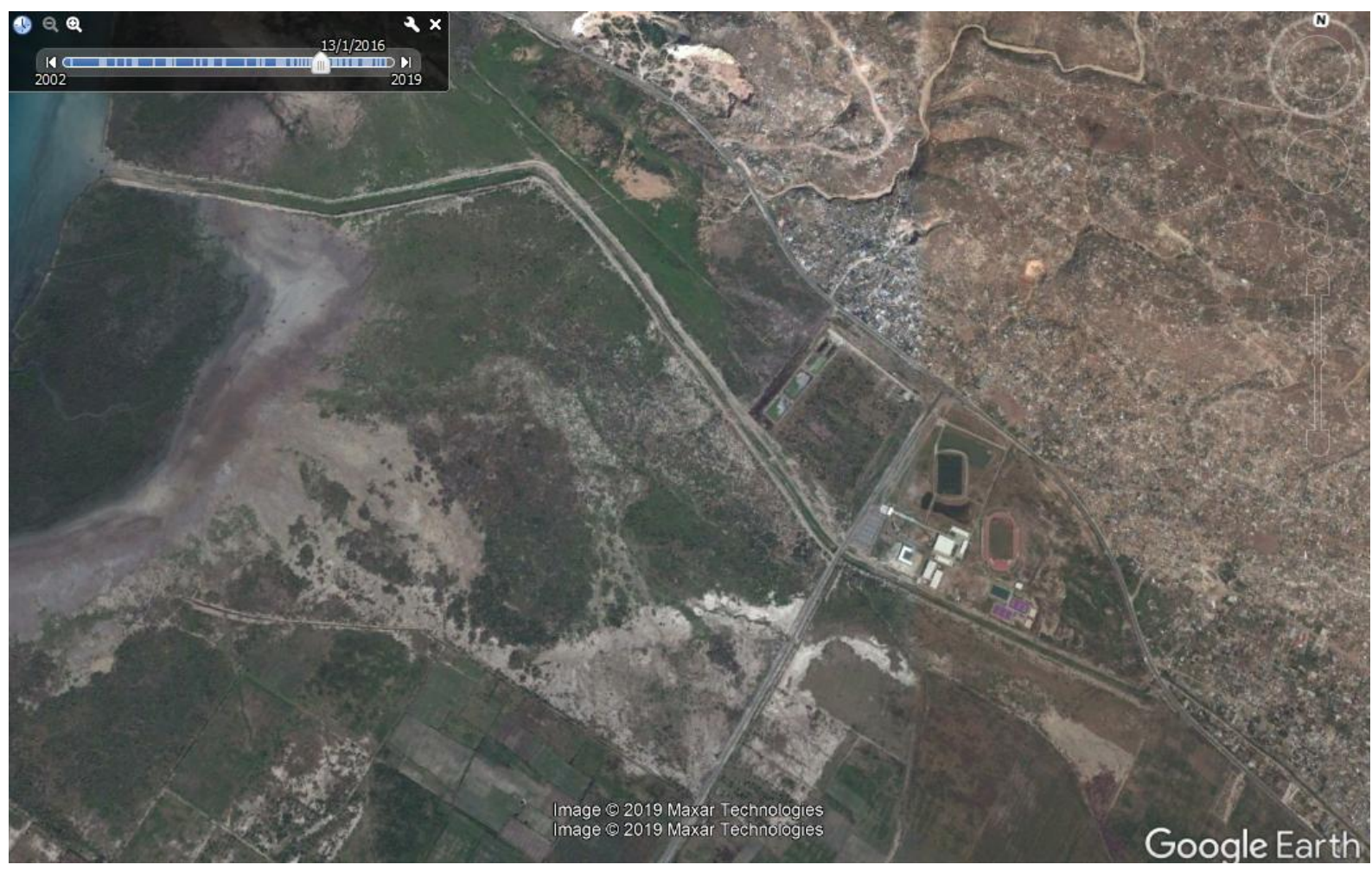

Localisation de la décharge sauvage « de Canaan ».

Plan schématique indiquant la situation de la décharge sauvage. Alice Corbet, 2020.

Grâce aux déplacés de Mosaïque avec qui je m'étais liée d'amitié et que je suivais dans leurs parcours d'habitats, et grâce à Willy, à qui je louais les services de chauffeur et qui, rapidement, est devenu un vrai assistant de recherche tant il m'ouvrait les portes de la compréhension du monde haïtien, j'empruntais quotidiennement la Route Nationale 1. A droite du ruban d'asphalte, Canaan et ses tentacules remplissaient progressivement les mornes vides. A gauche, la route flirtait avec le fil turquoise de la mer Caraïbe. Des marais tristes s'épanchaient jusqu'à cet horizon d'eau, occupés par des mangliers (arbustes dont les racines semblent de multiples échasses) et par les traces du canal Boucan-Brou qui se noyait en eux. Reliant l'Etang Saumâtre, à l'Est de la Plaine de Cul-de-Sac, au Golfe de la Gonâve, où se niche l'agglomération Port-au-Princienne, ce canal fut créé au XVIIIème siècle pour irriguer les plantations des sucreries alors installées par les colons (Cauna 2003, p.38). Si les traces de ces «habitations » sont aujourd'hui presque invisibles, ce canal rappelle le passé esclavagiste de la région. Toute cette zone à l'Ouest de la route 1, traversée par l'histoire d'Haïti, une nature malmenée, et l'abandon général, était uniquement tranchée par la perpendiculaire «Route Soleil 9 » (dite «Boulevard des Américains ») qui mène à Cité Soleil, quartier tristement célèbre de violence car soumis à la densité urbaine, à la pauvreté, aux affrontements de gangs, aux inondations, et car sa position côtière en fait le réceptacle des pollutions qui viennent de la ville en amont. C'est à Cité Soleil que la principale décharge de la capitale haïtienne, Truitier, fut créée en 1981.

Juste avant la Route Soleil 9, le «Stade Olympique pour l'Espoir» imposait ses infrastructures encore fraîches. Inauguré en juillet 2014, ce complexe sportif n'avait alors pas de gradins et était loin de ressembler aux images publicitaires initiales. Toutefois, il réunissait plusieurs pistes d'athlétisme et des associations sportives. Un peu incongru le long de la route, entre mer et Canaan, ce stade était un nouveau point de repère pour ceux qui empruntaient la route menant au Nord : il signalait la sortie de l'agglomération port-au-princienne. Mais juste 
après la route de Cité Soleil, des déchets amoncelés pourrissaient au soleil. Des camionspoubelles, parfois, traversaient ces empilements indistincts de sacs et de gravats. Sous la chaleur écrasante, se déplaçaient au milieu de tous ces fatras (terme créole pour « déchets ») des ombres qui m'intriguèrent: beaucoup d'enfants, qui arpentaient cet immense lieu d'abandon.

\section{Du labeur quotidien des chiffonniers}

Alors un jour, avec l'accord de Willy qui jugeait que le danger y était faible, et accompagnée par lui, nous nous arrêtâmes. Il ne doit pas exister de méthode pour entrer dans des terrains de misère aussi crue, ou je ne la connaissais pas. Je me suis contentée d'avancer un peu, essayant de ne pas poser mes pieds sur des objets tranchants. Ce jour-ci, c'était calme, les camions-poubelles n'étaient pas là. Ce sont des adolescents qui sont venus me voir, uniquement des garçons, regard froid, pas spécialement intrigués, continuant de fouiller à main nue ou avec un bâton le sol de leur domaine. «Je ne suis pas journaliste », ai-je tout de suite annoncé : ces derniers sont mal-perçus car les Haïtiens savent qu'ils donnent trop souvent une mauvaise image d'Haïti, mais aussi car ils ne veulent pas que des photos d'eux soient prises sans acquiescement ou pose. J'ai balbutié quelques mots de créole. Willy a expliqué que je venais tous les jours à Canaan, en face, et souhaitais juste comprendre comment fonctionnait cette décharge, par curiosité.

Quatorze fois entre 2012 et 215, je suis revenue à la décharge, sans jamais rester plus de 20 minutes, en posant peu de questions. Ce n'était pas toujours les mêmes garçons qui étaient là. Parfois, des adultes manifestaient leur mécontentement et il fallait partir. Parfois une indifférence feinte m'accueillait, parfois c'était cris et bousculades quand un camionpoubelles chargé à en déborder arrivait. Les camions étaient les trésors : il fallait être les premiers à ouvrir les sacs qu'ils libéraient, y trouver de quoi nourrir son corps, habiller sa vie. Alors, tous les coups étaient permis : l'arrivée des camions provoquait des courses effrénées et des guerres, parfois armées de contenants plus ou moins fonctionnels pour enfouir les trouvailles (seaux, sacs, paniers). 


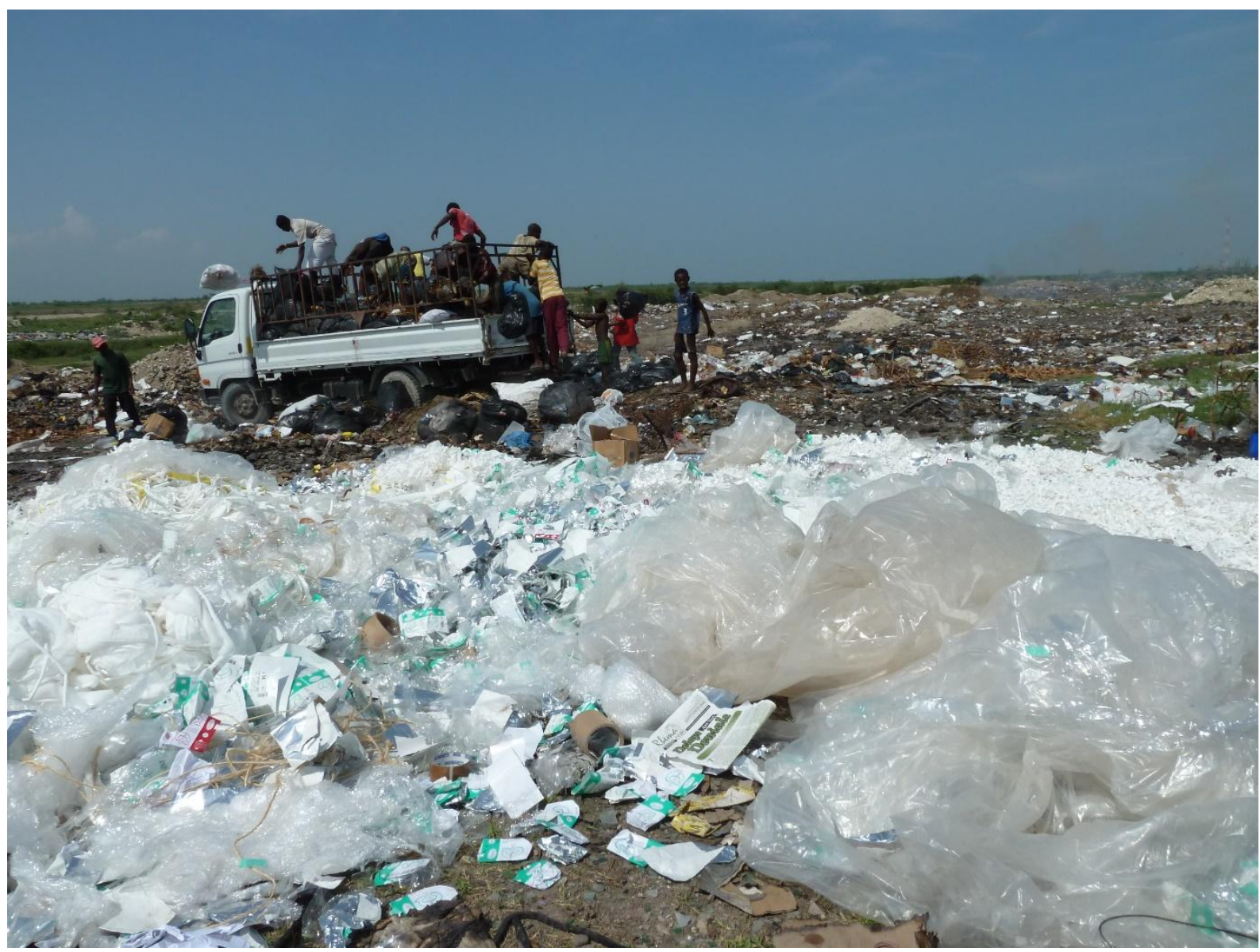

Arrivé quelques minutes auparavant, ce camion a déjà été vidé et les chiffonniers débutent le travail de tri.

Alice Corbet, septembre 2012

Les plus jeunes (dont j'estimais la moyenne d'âge autour de 12 ans) fonctionnaient en équipe. Deux principales s'opposaient parfois, au risque de faire tomber l'un d'entre eux sous les roues. Plusieurs tactiques s'affrontaient : suivre le camion au plus près pour y récupérer les sacs qui tombaient ou étaient jetés (les camions ne s'arrêtaient jamais), essayer de tirer sur le côté les ordures, ou porter un plus jeune jusqu'au sommet de la cargaison pour qu'il lance les déchets en contrebas. Les combats de coude s'engageaient sur le camion mais, une fois aux sols, les fatras appartenaient à leurs découvreurs. La plupart du temps, les sacs étaient déjà éventrés et l'exploration commençait, sans d'autre méthode que l'espérance de ce qu'ils pouvaient cacher.

Des sacs plastiques étaient sortis pour recueillir de la nourriture. Tout tissu était isolé avant d'être trié : encore utilisable comme habit, récupérable pour consolider un abri, ou définitivement à jeter. Les objets avaient un intérêt particulier, chacun tentant de comprendre à quoi pouvaient servir des éléments cassés ou inconnus : au pays des récupérateurs, l'imagination et le bricolage sont synonymes de survie. Parfois, des camions déposaient des denrées identiques, sans doute venues d'entreprises qui voulaient se débarrasser de leurs déchets ou malfaçons: un jour, arriva une cargaison de cartons abimés mais encore potentiellement exploitables pour faire du feu ou être transformés en objets de papiers mâchés. Un autre, je rencontrais un homme âgé qui triait patiemment une pile de papiers à cigarettes blanc, jetés car mal découpés, afin de les revendre à l'unité. Mais ce que tous recherchaient, et ce qui pouvait apporter autant de bonheur que de malheur (car ils 
provoquaient la convoitise), c'était des biens précieux. Un bijou, par exemple. Une croix en argent. Une poupée de plastique américaine. Tout ce qui peut se revendre, toute plus-value en dehors des usuels résidus jetés par les vies urbaines. Les contenants en plastique avaient une vraie valeur pratique, et les métaux pouvaient facilement être revendus ou échangés : le fer, souvent «mis à nu» lors du brûlage des tas d'immondice (d'où de réels risques toxiques ou de brûlures), pouvait ensuite être réutilisé et réapparaître sous forme d'objets divers ou de matériaux de construction. Chaque matière triée était rassemblée dans de gros ballots de tissus, et je n'ai jamais vu qui venait les chercher. 


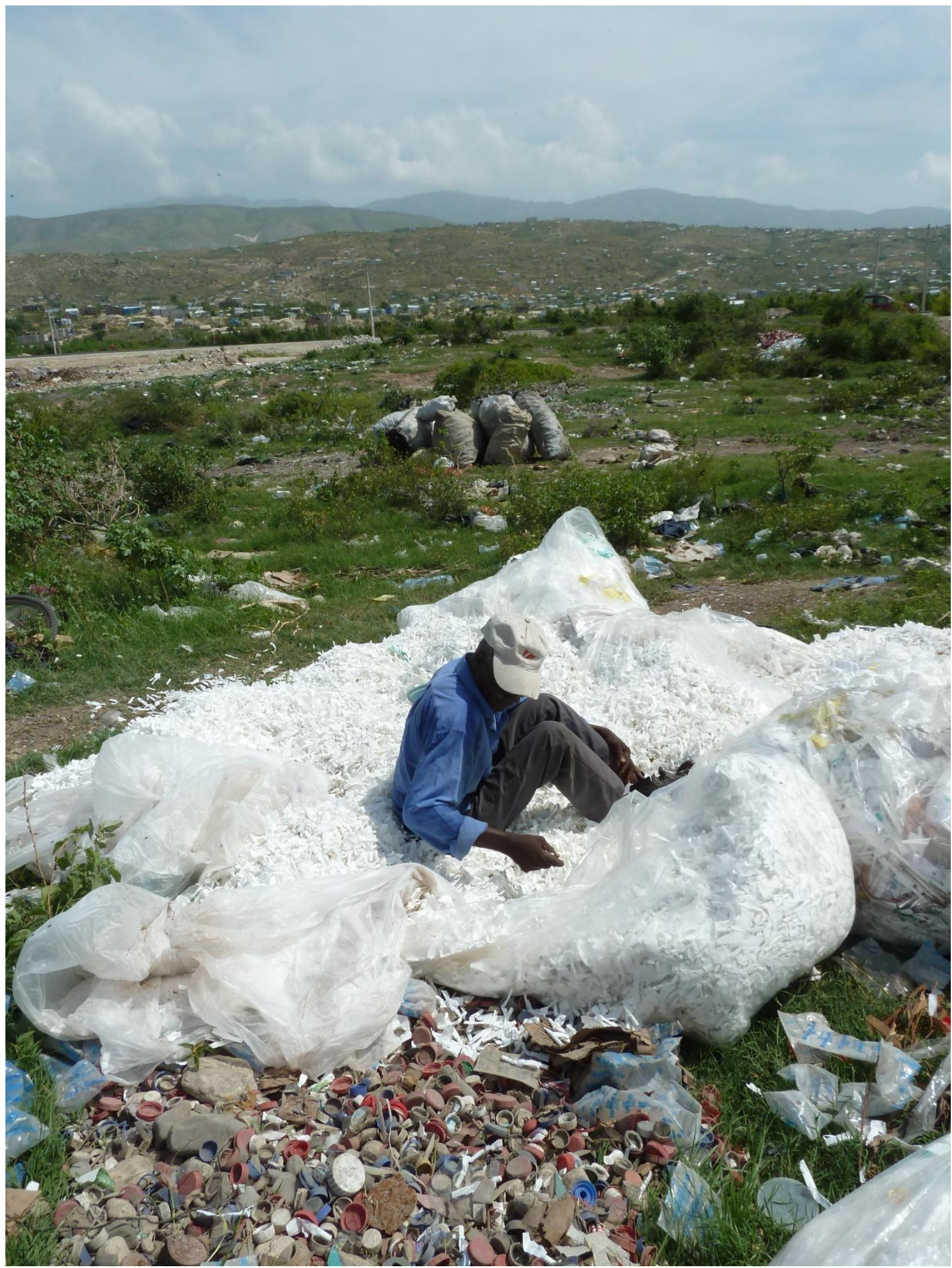

Plus âgé et donc exclu de la « courses » quand les camions arrivent, cet homme a récupéré des papiers de cigarette jetés pour malfaçon et les trie avant une revente au détail.

Alice Corbet, septembre 2012

Les trouvailles étaient partagées avec le groupe. Un chef, le plus âgé -j'estimais son âge aux alentours de 15 ans-, récupérait les plus belles prises. Il s'attachait toutefois à 
redistribuer quelques biens à ceux qui les avaient trouvés. Son rôle était d'organiser la bande et d'essayer, ensuite, de revendre ce qui était possible. Un de ces adolescent plusieurs fois croisé, assez hostile, était lui-même redevable à quelques adultes. Ainsi, l'accès à la décharge était très contrôlé : c'était un territoire surveillé. D'ailleurs, quand le soleil tombait, les jeunes chercheurs de trésors n'habitaient pas loin : ils étaient installés dans des cahutes, juste de l'autre côté de la route nationale 1. Cela leur permettait, le jour, de proposer leurs trouvailles à quelques automobilistes intéressés. Leur accès à l'eau était compliqué, mais l'accroissement de Canaan leur permettait de pouvoir aller boire plus facilement. Pour la toilette, la mer Caraïbe n'était pas si loin. Et Cité Soleil un lieu de ressources si nécessaire.

\section{La décharge « de Canaan »}

Cette décharge n'a pas de nom officiel. On m'a dit, en 2012, que c'était celle de «Titanyen », du nom d'une petite ville située le long de la route, plus au Nord. Triste référence à l'endroit connu pour avoir été le lieu des exécutions lors des dictatures duvaliéristes (1957-1986). Quand quelqu'un « disparaissait », à cette époque, on allait faire un tour du côté de Titanyen, pour vérifier si on ne retrouvait pas son corps... Par la suite, Titanyen a été le lieu choisi pour creuser les fosses communes nécessaires à l'enterrement de tous les morts du séisme qui ne pouvaient l'être dans des cimetières, soit qu'ils n'aient pas été identifiés, soit que leurs familles ne pouvaient leur offrir une sépulture (Corbet, 2014).

Plus certainement, la décharge se situe dans la section communale «Les Varreux 1 » de Croix-des-Bouquets, au lieu-dit «Sources puantes », nom peu avenant expliqué par la présence d'une source d'eau chaude qui dégage une odeur soufrée désagréable, mais dont les vertus sont vantées. Son accès difficile néanmoins, entre décharge et marais, rend le site plus connu de réputation que d'usage. La nuit, dit-on, des pratiques vaudous y ont lieu, pour bénéficier des aspects thérapeutiques comme magiques de l'eau (le vaudou estime que l'eau peut être l'expression des loas (les esprits), et notamment de Agwé, loa de la mer et des pêcheurs) : l'eau est souvent utilisée dans des remèdes curatifs ou des sortilèges).

Cette décharge est surtout illégale, et ceux qui venaient y déverser leurs ordures me montraient bien qu'ils ne souhaitaient pas que j'interagisse avec eux. Pourquoi venir ici ? Sans doute parce que l'accès est facile. Sans doute aussi que son illégalité dispense les propriétaires des camions des démarches associées à Truitier, la décharge officielle. Cette dernière est pourtant située non loin, aux flancs de Cité Soleil, d'une ravine (celle de la Rivère Grise) et de la mer où beaucoup de déchets terminent leur vie. C'est le Service Métropolitain de Collecte des Résidus Solides (SMCRS) qui s'occupe du ramassage dans les huit communes de l'agglomération de Port-au-Prince (dont la population est estimée à plus de 3 millions d'habitants), ainsi qu'une dizaine de compagnies privées. A Truitier, les entreprises et particuliers qui viennent verser leurs déchets doivent payer à la commune de Cité Soleil des taxes pour financer la gestion de leurs détritus. Or, ces taxes ne sont jamais restituées sous forme d'achat de matériel, de protection des travailleurs, ou d'assainissement des lieux. Elles sont en outre considérées comme trop élevées par les entreprises. Pas étonnant, alors, que sur une quantité moyenne de déchets estimée à six mille $\mathrm{m} 3$ par jour à Port-au-Prince, seulement $30 \%$ sont collectés : «le reste est déversé notamment dans les ravins et les rues » (Milfort 2018) ou dans des décharges sauvages telles que celle que je découvrais. Dans un état des lieux sur la gestion des déchets dans la capitale suite au séisme, des chercheurs rappellent effectivement que «une partie des déchets échappe au contrôle des autorités. Ils sont soit brûlés, soit évacués vers des espaces vides (terrains vagues, berges de cours d'eau, etc.), soit valorisés par tout un circuit informel d'acteurs » (Popescu, Durand, d'Ercole, 2014). 
Celle qui est aujourd'hui qualifiée de «décharge de Canaan » préexistait pourtant au camp qui s'est installé de l'autre côté de la route. Nul doute qu'elle continue de se développer grâce à lui. On note alors que cette décharge sauvage, qui était auparavant un lieu de relégation dans un espace «libre » mais éloigné des habitations, a été rattrapée par la densité urbaine pour se transformer en décharge ordinaire dans un pays où, comme c'est souvent le cas dans les villes pauvres, se côtoient les logiques spatiales de relégation (éloigner le déchet) et de proximité (car il est compliqué de le transporter trop loin) (Florin, Cirelli 2015). L'observation démontre que la grande majorité des ménages des quartiers précaires de la capitale déversent leurs déchets dans les ravines ou les décharges sauvages, car ils n'ont pas d'autre lieux officiels ni de systèmes de ramassage organisé (Bras 2010, p.155). La décharge de Canaan est donc devenue le lieu de rejet «naturel » pour le nouveau quartier limitrophe, comme si elle validait ainsi tant son existence que sa précarité.

\section{Un lieu de marginalisation et de craintes}

Bien sûr, le déchet de l'un alimente souvent le mode de vie de l'autre : la notion de « déchets » est donc très subjective (Drackner 2005). Mais l'illégalité de la décharge de Canaan marginalise doublement les personnes qui y travaillent. Manipuler des déchets, c'est porter une souillure, au sens de Mary Douglas (Douglas 1966) : l'activité en elle-même, bien que nécessaire, est mal perçue et non valorisante, et exclut socialement celui qui la mène. Lhuilier et Cochin ont renchérit en insistant sur le fait que les récupérateurs valorisent le déchet en lui évitant de disparaître, mais que ces derniers entachent en même temps ceux qui le manipulent, comme par contamination (Lhuiller Cochin 1999). En outre, les travailleurs de la décharge ne sont pas protégés par une organisation à minima, même dysfonctionnant, comme celle de Truitier qui essaie de structurer le travail, de mieux l'organiser, et de mieux insérer les travailleurs dans le panorama social. Les jeunes qui travaillent «à Canaan » répondent à la loi du plus fort. Ce sont des kokorat : ce terme créole péjoratif désigne à la fois un animal rampant, souvent trouvé dans les ordures, et des personnes «en dessous », sales ou mal agissantes. Par extension, il qualifie les enfants des rues et les ramasseurs des déchets. Les croyances associées aux effets personnels relèguent aussi les travailleurs des décharges à un rang à part : dans le vaudou, les objets, et surtout les habits où ce qui a été manipulé quotidiennement par quelqu'un, gardent une «trace » de leur propriétaire. Cette dernière peut être récupérée dans le cadre de magie noire : il faut donc la perdre, l'éloigner (Hurbon 2002). Les craintes qu'inspirent ceux qui manipulent les effets abandonnés ne sont donc pas neutres et contribuent à leur marginalisation.

Sur la petite dizaine de chiffonniers à qui j'ai pu poser des questions, seulement deux avaient encore un parent isolé. Certains connaissaient leurs frères et sœurs, sans être sûrs des liens de sang qui les unissaient : ils évoluaient au sein d'une «bande » depuis des années. Leurs réseaux étaient ceux des enfants des rues, ce qui contribuait à expliquer que le système de revente des produits récoltés soit aussi anarchique : chacun tentait de cacher ce qu'il avait ou alors de le remettre à ses proches, et ceux qui supervisaient l'ensemble, invisibles à mes yeux mais disciplinant la décharge de leur autorité, semblaient davantage faire partie de réseaux violents qui instrumentalisent les enfants des rues que de compagnies privées qui dévoyaient leurs actions. Ainsi, un adolescent me montrait avec fierté des médicaments anonymes qui allaient être revendus à l'unité, sur les bords des routes, par des réseaux illicites détaillant des produits avariés ou aux origines douteuses. 
Les récupérateurs des décharges ne sont pourtant pas à la première étape de la chaîne. Auparavant, une grande partie des déchets est déjà filtrée par les familles elles-mêmes, qui savent user au mieux des rebus, puis par les nombreux mendiants ou ramasseurs qui fouillent les poubelles, sacs et éventuelles bennes dans la ville. Le parcours des objets est donc difficile à suivre, mais ils mettent du temps avant de réintégrer le secteur formel, par exemple pour être retransformés à plus grande échelle. Ils demeurent alors dans le cadre d'usages récurrents dans l'informalité haïtienne : la «débrouille», telle qu'elle est nommée en créole. Ces différentes chaînes de récupérations n'en demeurent pas moins des éléments importants dans l'économie urbaine et sociale qui pallie les déficiences étatiques.

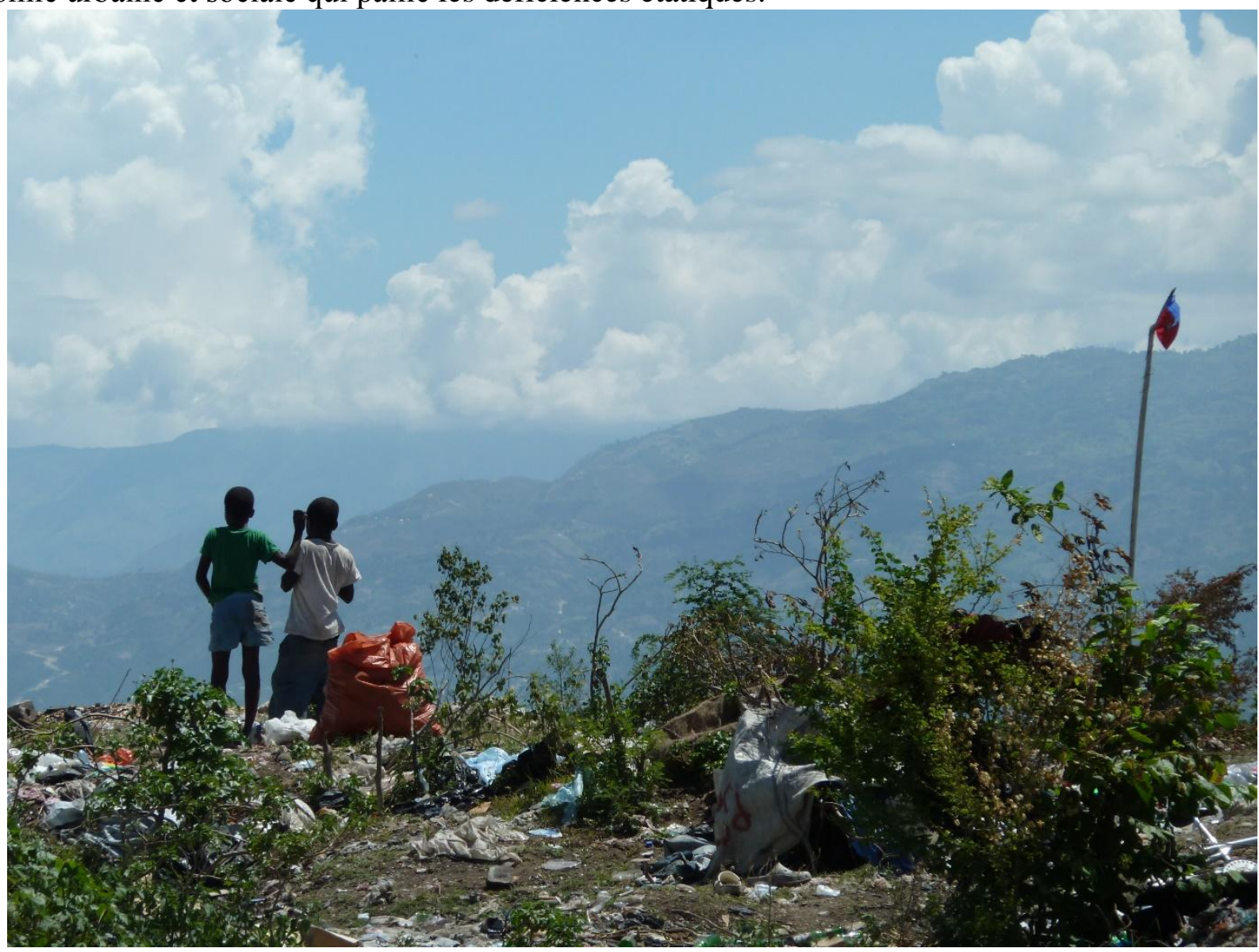

Un drapeau haïtien a été dressé près des ballots de matières récupérées par les jeunes chiffonniers. Au fond, les mornes où s'étend le camp-ville de Canaan.

Alice Corbet, septembre 2012

\section{L’impasse méthodologique et émotionnelle}

J'aurais pu continuer à travailler sur cette décharge. Mais j'ai préféré privilégier Canaan, cet immense bidonville de l'autre côté de la route, d'abord stigmatisé car destiné aux sans-abris, puis devenu au fil des années une ville organisée, investie, développée. Canaan m'attirait parce qu'on y construisait; à l'inverse, la décharge était un lieu de destruction. Il fallait aussi y supporter la misère, dans tout ce qu'elle a de plus cru, trivial : les odeurs, les carcasses d'animaux en putréfaction, les réflexes de rejet physique devant le lixiviat (le « jus » de poubelles), la tristesse d'assister à la pollution du sol, de l'air, de la mer, notamment quand des produits toxiques encombraient la décharge; et la violence subie, mais aussi exprimée, par les personnes y travaillant. J'atteignais alors une limite à mes capacités de travail : aurais-je vraiment réussi à mener une enquête ici ? Comment réussir à aborder, 
quotidiennement, ces éléments physiques rebutants, comment les dépasser pour faire place à une recherche anthropologique de qualité ? Comment sortir des sentiments contradictoires, entre crainte, dégoût, empathie, intérêt, qui se croisent intensément dans ce climat spécifique ? Certains chercheurs réussissent à mener des enquêtes ethnographiques sur de tels terrains (Le Lin, Harpet, 2003), comme certains dans des abattoirs (Vialles, 1987) : ce n'est pas mon cas.

Alors que Bauman parle des « déchets humains » qui se retrouvent dans les marges, en évoquant les réfugiés et les personnes ghettoïsées (la couverture de son fameux ouvrage «Vies perdues » laisse deviner, en ombre chinoise, un enfant déambulant au milieu d'immondices) (Bauman 2004), le travailleur des décharges est toujours au centre d'un réseau, même marginalisé, et utilise le déchet pour vivre. Il est difficile de parler de lui sans le misérabilisme qui est souvent associé à sa condition. Il est difficile, également, de ne pas tomber dans un discours héroïsant ceux qui, chaque jour, œuvrent dans les détritus. Haïti, Cité Soleil et Truitier sont déjà trop souvent associés à des images noires, choquantes de pauvreté et de violence sociale ou physique, qui sont diffusées dans le monde entier et contribuent à associer le pays à un lieu d'exclusion et d'horreur. Beaucoup trop de photographies rapportées de Haïti soulignent la misère, voire l'esthétisent, comme la célèbre photo de Alice Smeets montrant une jeune fille traversant un océan de détritus sur fond de bidonville qui reçut le prix Unicef en 2008. Pour ma part, encombrée par les questions méthodologiques exposées auparavant, je ne souhaitais pas non plus diffuser des images qui stigmatisent Haïti auprès d'étrangers qui oublient souvent que leurs propres rebuts sont parfois envoyés sous d'autres contrées, ou qu'ils font vivre de nombreux chiffonniers aux pieds de leurs immeubles (Oliveira 2015) ${ }^{1}$. Mais je me rappellerai toujours d'une image emblématique de la décharge de Canaan : un drapeau haïtien, perché en haut d'un bâton, un peu à l'écart des déchets. La décharge, alors, était fière, et citoyenne.

\section{Bibliographie :}

- Bauman Z. , 2004. «Wasted lives. Modernity and its Outcasts ». Cambridge: Polity, $152 \mathrm{p}$.

- Bras A. , 2010. Eléments pour une définition de la problématique de la propreté urbaine en Haïti : Le cas de Port-au-Prince. Thèse de doctorat en Géographie, aménagement, urbanisme, sous la direction de E. Evens, cotutelle de L'Institut National des Sciences Appliquées de Lyon (France) et de l'Université Quisqueya (Haïti).

- Cauna, J. de, 1987. Au temps des îles à sucre: Histoire d'une plantation de SaintDomingue au XVIIIe siècle., Paris, Karthala, 285 p.

- Cirelli C. , Florin B. , Bercegol R. , 2019. «La mise en image du rebut ». EchoGéo, numéro 47. https://journals.openedition.org/echogeo/17103

- Corbet A. , 2014. "Invisibles omniprésents, les morts du séisme », in L. Hurbon, Catastrophes et environnement, Paris, Editions de l'EHESS, p. 29-58.

- Corbet A. , 2015. "Community after all? An inside perspective of encampment in Haiti ». Journal of Refugee Studies, volume 29, n², p. 166-186.

- Douglas M. , 1966. Purity and Danger. An Analysis of Concepts of Pollution and Taboo. Londres: Routledge and Kegan Paul, 196 p.

\footnotetext{
${ }^{1}$ Une exposition « La mise en image du rebut », réalisée par les chercheurs du réseau Sociétés urbaines et déchets, expose des images de récupérateurs autour du monde avec un regard différent, « débarrassés du stigmate qui accompagne le contact avec l'ordure » (Cirelli, Florin, Bercegol, 2019).
} 
- Drackner M. , 2005. «What is waste? To whom?--An anthropological perspective on garbage ». Waste Management and research, volume 23, numéro 3, p. 175-181.

- Florin B., Cirrelli C. (dir.), 2015. Sociétés urbaines et déchets : éclairages internationaux. Presses Universitaires François Rabelais, 452p.

- Hurbon L. , 2002. Dieu dans le vaudou Haïtien. Paris, Maisonneuve et Larose, 268p.

- Le Lin B. , Harpet C. , 2003. Vivre sur la décharge de Antananarivo. Regards anthropologiques. Paris, L'Harmattan, 2003, 240.

- Lhuillier D. , Cochin Y. , 1999. Des déchets et des hommes. Paris, Desclée de Brouwer, $185 \mathrm{p}$.

- Milfort M, 2018, «A qui profite la mauvaise gestion de la décharge de Truitier ». Haiti Liberté : https://haitiliberte.com/a-qui-profite-la-mauvaise-gestion-de-ladecharge-de-truitier/

- Olivera M. , 2015. «Insupportables pollueurs ou recycleurs de génie ? Quelques réflexions sur les «Roms » et les paradoxes de l'urbanité libérale », Ethnologie française, volume 3, numéro 153), p. 499-509.

- Popescu R., Durand M. , d'Ercole R. 2014., « La gestion des déchets post-catastrophe à Port-au-Prince: entre relégation et proximité »,EchoGéo, numéro 30. http://journals.openedition.org/echogeo/14070

- Vialles N., 1987. Le sang et la chair. Les abattoirs du pays de l'Adour. Paris, Editions de la Maison des Sciences de l'homme, 162p. 